\title{
Plasmonic Structure Enhanced Exciton Generation at the Interface between the Perovskite Absorber and Copper Nanoparticles
}

\author{
Sheng Hsiung Chang, ${ }^{1}$ Kuen-Feng Lin, ${ }^{2}$ Chien-Hung Chiang, \\ Sheng-Hui Chen, ${ }^{2}$ and Chun-Guey $\mathrm{Wu}^{1}$ \\ ${ }^{1}$ Research Center for New Generation Photovoltaics, National Central University, No. 300 Jhongda Road, Jhongli, \\ Taoyuan County 32001, Taiwan \\ ${ }^{2}$ Department of Optics and Photonics, National Central University, No. 300 Jhongda Road, Jhongli, Taoyuan County 32001, Taiwan
}

Correspondence should be addressed to Sheng Hsiung Chang; shchang@ncu.edu.tw,

Sheng-Hui Chen; ericchen@dop.ncu.edu.tw, and Chun-Guey Wu; t610002@cc.ncu.edu.tw

Received 30 June 2014; Revised 5 August 2014; Accepted 19 August 2014; Published 11 September 2014

Academic Editor: Rui C. Marques

Copyright (c) 2014 Sheng Hsiung Chang et al. This is an open access article distributed under the Creative Commons Attribution License, which permits unrestricted use, distribution, and reproduction in any medium, provided the original work is properly cited.

The refractive index and extinction coefficient of a triiodide perovskite absorber (TPA) were obtained by fitting the transmittance spectra of TPA/PEDOT:PSS/ITO/glass using the transfer matrix method. Cu nanoplasmonic structures were designed to enhance the exciton generation in the TPA and to simultaneously reduce the film thickness of the TPA. Excitons were effectively generated at the interface between TPA and Cu nanoparticles, as observed through the 3D finite-difference time-domain method. The exciton distribution is advantageous for the exciton dissociation and carrier transport.

\section{Introduction}

In recent years, mixed halide $\left(\mathrm{CH}_{3} \mathrm{NH}_{3} \mathrm{PbI}_{3-x} \mathrm{Cl}_{x}\right)$ and triiodide $\left(\mathrm{CH}_{3} \mathrm{NH}_{3} \mathrm{PbI}_{3}\right)$ perovskite absorber (PA) based photovoltaics have been intensively investigated because a power conversion efficiency (PCE) of $15 \%$ can be achieved by solution processes under low temperatures $[1,2]$. There are several factors that can explain the high PCE. The bandgap of PA is about $1.64 \mathrm{eV}$ [3], which can absorb half of the sun light. The exciton diffusion length of PA is longer than 1 micrometer [4]; therefore the bilayered structure based photovoltaics are workable [1-4]. The exciton binding energy of PA is about $50 \mathrm{meV}$ [5], which results in good exciton dissociation at the interface between the PA and PCBM (Spiro-OMeTAD) [4]. The sharp optical absorption edge of PA corresponds to the small Urbach energy ( $15 \mathrm{meV})$, which results in a high fill factor [6]. The thickness of PA has to be $\sim 400 \mathrm{~nm}$ in order to efficiently absorb the incident sun light. However, a thicker PA is disadvantageous for exciton dissociation and carrier transport, limiting the photovoltaic performances in terms of short-circuit current density and fill factor. A PCE as high as $20 \%$ can be expected by improving the fill factor [7]. The above-mentioned drawbacks can be improved by using nanoplasmonic structures [8, 9]. Two degenerate transverse plasmon modes are supported by twodimensional ordered $\mathrm{Cu}$ nanoplasmonic structure embedded in P3HT:PCBM blended film, which has been designed to enhance the absorption of P3HT:PCBM based inverted photovoltaics by $22 \%$ in the visible range [9]. In this work, the $\mathrm{Cu}$ nanoplasmonic structures were used to enhance exciton generation in the triiodide perovskite absorber (TPA) while simultaneously reducing the film thickness of TPA. The transfer matrix method (TMM) was used to calculate the transmittance, reflectance, and absorptance. The 3D finitedifference time-domain (FDTD) method was used to observe the plasmon-mediated exciton generation. 


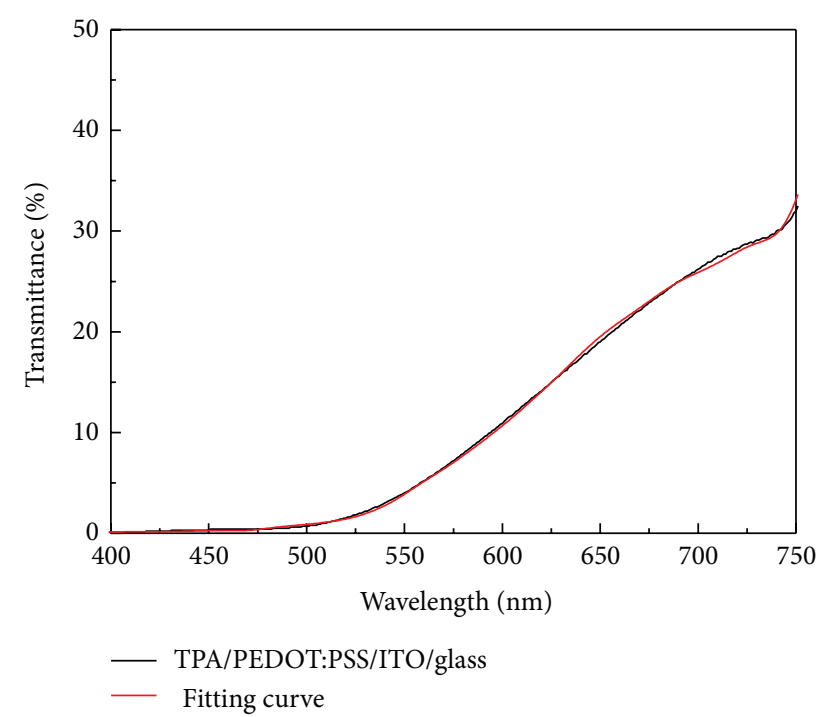

(a)

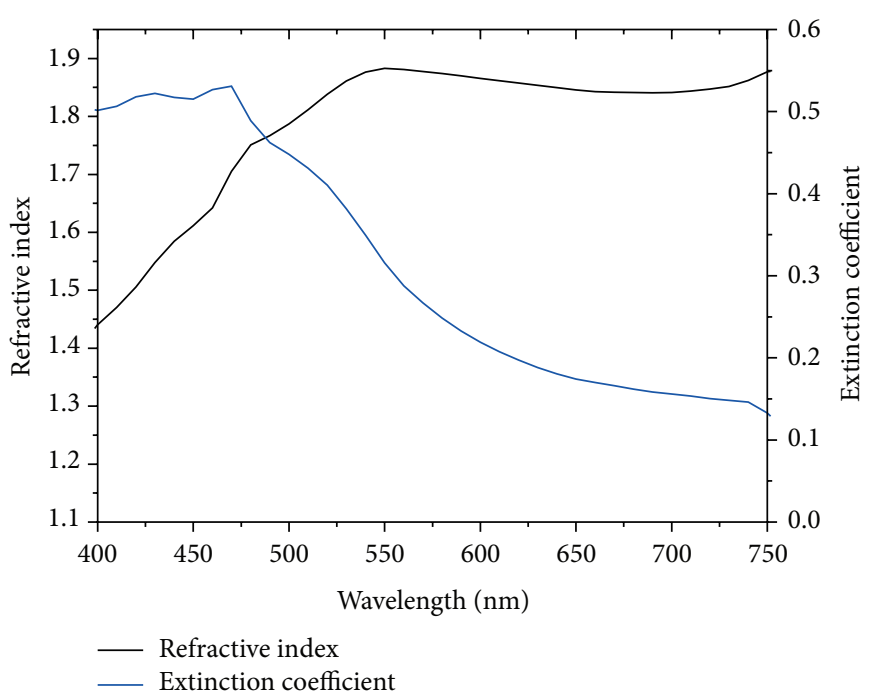

(b)

FIGURE 1: (a) Transmittance spectra of TPA/PEDOT:PSS/ITO/glass under normal incidence. (b) Refractive index and extinction coefficient of TPA film.

\section{Optical Constants of Triiodide Perovskite Absorber}

TPA was spin-coated on top of the PEDOT:PSS/ITO/glass by a sequential deposition method [1]. Figure 1(a) presents the transmittance spectrum of the TPA/PEDOT:PSS/ITO/glass. The transmittance spectrum was measured by a high accuracy spectrometer (Hitachi U-4100). The film thickness for each layer was measured by an $\alpha$-step device (Veeco Dektak 150). The thicknesses of the TPA, PEDOT:PSS, and ITO were found to be $400 \mathrm{~nm}, 20 \mathrm{~nm}$, and $250 \mathrm{~nm}$, respectively. The refractive indices and extinction coefficients of the PEDOT:PSS thin film and the ITO film were taken from [9]. A Lorentz model was used to describe the dielectric constant of TPA, which can be written as follows:

$$
\varepsilon_{\mathrm{TPA}}(\omega)=\varepsilon_{b}+\sum_{n=1}^{14} \frac{s_{n} \omega_{L, n}}{\omega_{L, n}^{2}-\omega^{2}-j G_{n} \omega},
$$

where $\varepsilon_{b}(=1.5)$ is the background dielectric constant, $n$ is the $n$th Lorentz pole, $s_{n}$ is the strength, $\omega_{L}$ is the oscillation frequency, and $G$ is the decay rate. Fourteen Lorentz oscillators were used in the fitting process. The transmittance spectrum of the TPA/PEDOT:PSS/ITO/glass was fitted using TMM. The fourteen oscillating wavelengths " $\lambda_{L}$ " were fixed and are listed in Table $1 . \lambda_{L}=2 \pi c / \omega_{L}$, where $c$ is light speed in vacuum. The oscillation strengths and decay rates were scanned in the fitting process. An error function (EF) is defined to evaluate the accuracy between fitting and experimental curves. Consider

$$
\mathrm{EF}=\sum_{\lambda=400}^{750} \frac{\left|T_{\text {fit }}(\lambda)-T_{\exp }(\lambda)\right|}{351 \times T_{\exp }},
$$

where $T_{\text {fit }}$ (red line) is the transmittance of the fitting curve and $T_{\exp }$ (black line) is the measured transmittance of the
TABLE 1: Fitted parameters of the Lorentz model for TPA film.

\begin{tabular}{lcc}
\hline $\begin{array}{l}\text { Oscillation wavelength } \\
(\mathrm{nm})\end{array}$ & Strength & $\begin{array}{c}\text { Decay rate } \\
\left(\times 10^{14} / \mathrm{s}\right)\end{array}$ \\
\hline$\lambda_{L, 1}=380$ & $s_{1}=0.2$ & $G_{1}=10$ \\
$\lambda_{L, 2}=430$ & $s_{2}=0.15$ & $G_{2}=8$ \\
$\lambda_{L, 3}=470$ & $s_{3}=0.1$ & $G_{3}=6$ \\
$\lambda_{L, 4}=500$ & $s_{4}=0.04$ & $G_{4}=4$ \\
$\lambda_{L, 5}=520$ & $s_{5}=0.035$ & $G_{5}=3.5$ \\
$\lambda_{L, 6}=540$ & $s_{6}=0.032$ & $G_{6}=3.5$ \\
$\lambda_{L, 7}=570$ & $s_{7}=0.03$ & $G_{7}=4$ \\
$\lambda_{L, 8}=600$ & $s_{8}=0.02$ & $G_{8}=4$ \\
$\lambda_{L, 9}=630$ & $s_{9}=0.02$ & $G_{9}=4$ \\
$\lambda_{L, 10}=670$ & $s_{10}=0.02$ & $G_{10}=3.5$ \\
$\lambda_{L, 11}=710$ & $s_{11}=0.015$ & $G_{11}=3$ \\
$\lambda_{L, 12}=720$ & $s_{12}=0.008$ & $G_{12}=3$ \\
$\lambda_{L, 13}=730$ & $s_{13}=0.009$ & $G_{13}=2$ \\
$\lambda_{L, 14}=745$ & $s_{14}=0.01$ & $G_{14}=1.5$ \\
\hline
\end{tabular}

TPA/PEDOT:PSS/ITO/glass. The value of the error function is equal to 0.025 . The refractive index " $n$ " and extinction coefficient " $k$ " of the TPA film can be obtained by $n+$ $i k=\left(\varepsilon_{\mathrm{TPA}}\right)^{1 / 2}$. The optical constants of TPA are plotted inFigure 1(b). The fitted parameters are listed in Table 1.

\section{Plasmonic Structure Enhanced Exciton Generation}

Figure 2 presents the absorptance spectra of the TPA/ITO/glass, which was calculated using TMM. The absorptance of the TPA increases with an increase in thickness 


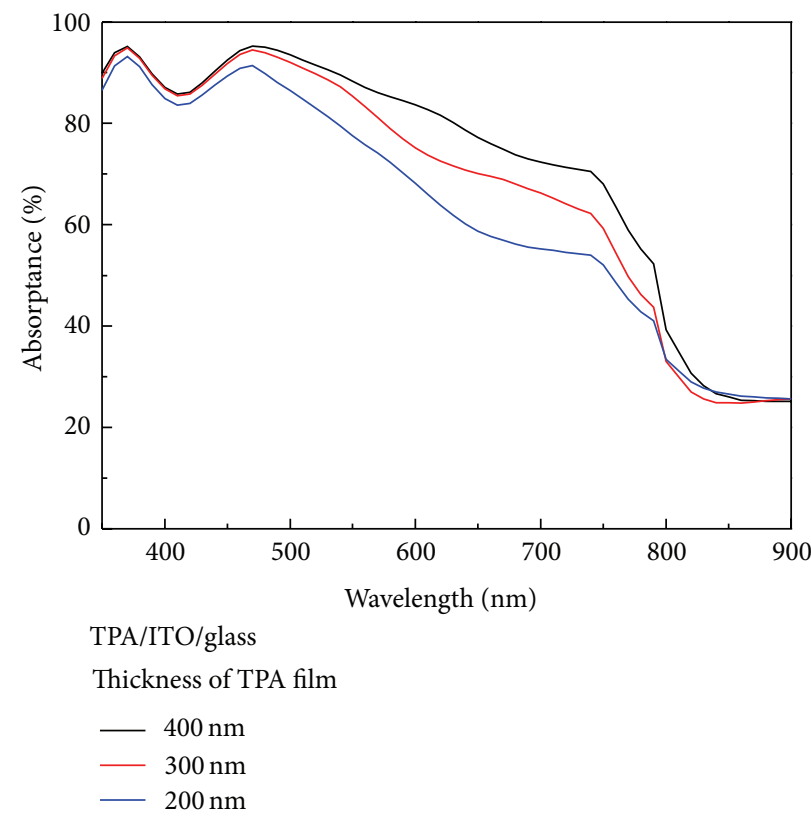

FIGURE 2: Absorptance spectra of TPA/ITO/glass for different TPA thicknesses.

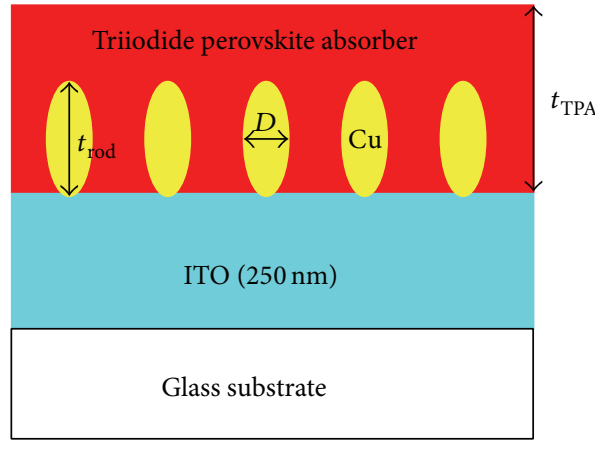

(a)

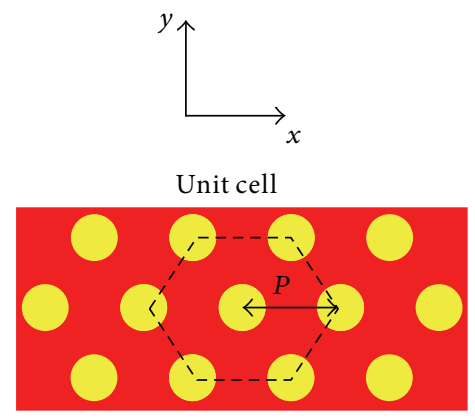

(b)

FIgURE 3: (a) Side view of TPA/ITO/glass with the Cu nanoplasmonic structure. (b) Top view of the Cu nanoplasmonic structure embedded in the TPA thin film.

from $200 \mathrm{~nm}$ to $400 \mathrm{~nm}$. In the wavelength range of $800 \mathrm{~nm}$ to $900 \mathrm{~nm}$, the incident near-infrared light is absorbed by the ITO film due to the free carrier absorption. In order to reduce the thickness, a $\mathrm{Cu}$ nanoplasmonic structure was embedded in the TPA in order to enhance the absorptance in the effective absorption range ( $350 \mathrm{~nm}$ to $760 \mathrm{~nm}$ ). Figure 3 presents the $\mathrm{Cu}$ nanoplasmonic structure embedded in the TPA thin film. In this study, the period " $P$ " was fixed at $100 \mathrm{~nm}$. The gap size is defined by the difference between the period and the diameter of $\mathrm{Cu}$ nanoparticles.

The dipole-coupling model (DCM) [10] was adopted to calculate the effective dielectric constant of $\mathrm{Cu}$ nanoplasmonic structure embedded in the TPA film. These nanoplasmonic structures embedded can be treated as an effective medium. The physical concept of DCM is described in [9].
The Lorentz-Drude model was applied to $\mathrm{Cu}$ to calculate the refractive index and absorption coefficient [11].

Figure 4 presents the absorptance spectra of the TPA with and without the $\mathrm{Cu}$ nanoplasmonic structures. There was an increase in the absorptance of the TPA with $\mathrm{Cu}$ nanoplasmonic structures when the gap was changed from $50 \mathrm{~nm}$ to $30 \mathrm{~nm}$. Compared with the red dashed line (TPA thickness $=200 \mathrm{~nm}$ ) in Figure 4, the absorptance indicated by the black line is larger because the transverse plasmonic (TP) mode enhances the absorption (exciton generation) of the TPA. Compared with the black dashed line (TPA thickness $=400 \mathrm{~nm}$ ) in Figure 4, the absorptance indicated by the black line is smaller. The absorptance spectra of a thicker TPA film with and without $\mathrm{Cu}$ nanoplasmonic structures are presented in Figure 5. Compared with 

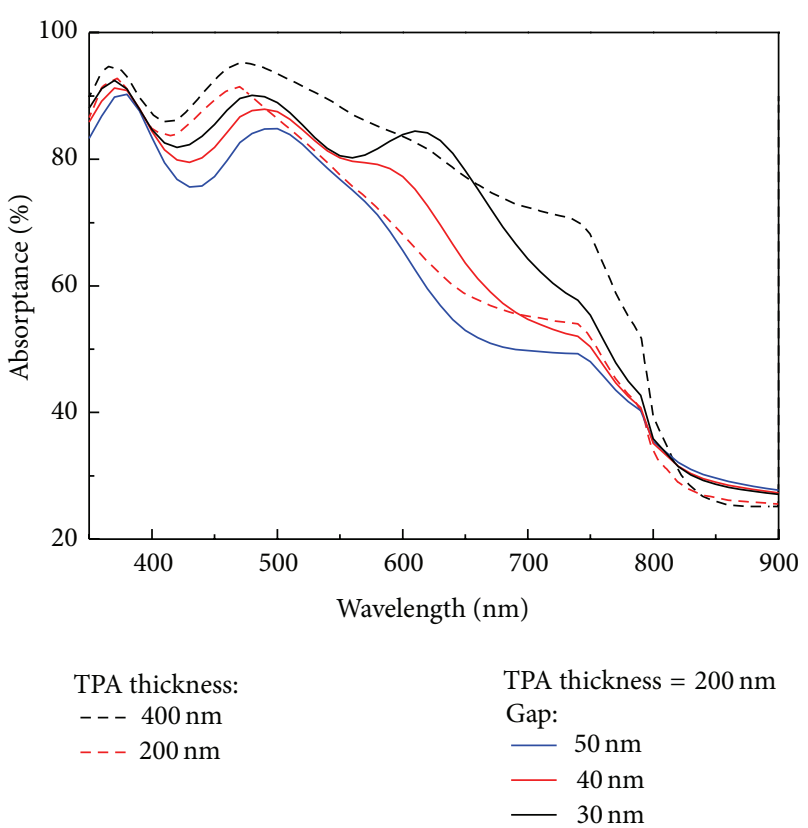

FIgURE 4: The red (black) dashed line indicates the absorptance of the TPA/ITO/glass when the TPA thickness is equal to $200 \mathrm{~nm}$ $(400 \mathrm{~nm})$. The blue, red, and black lines are the absorptance of the TPA/ITO/glass with the Cu nanoplasmonic structure when the long axis of the $\mathrm{Cu}$ nanoparticles is $100 \mathrm{~nm}$.

the black dashed line (TPA thickness $=400 \mathrm{~nm}$ ) in Figure 5, the absorptance indicated by the black line is higher due to the TP mode enhanced absorption even though the TPA thickness $(=300 \mathrm{~nm})$ is thinner. The $\mathrm{Cu}$ nanoplasmonic structure enhanced the absorptance of TPA in the effective absorption range $(350 \mathrm{~nm}-760 \mathrm{~nm})$ by $1.7 \%$ while reducing the TPA thickness from $400 \mathrm{~nm}$ to $300 \mathrm{~nm}$.

\section{Exciton Distribution}

The 3D FDTD method was used to calculate the electric and magnetic field distributions of the $\mathrm{Cu}$ nanoplasmonic structures embedded in the TPA film. 20-cell perfectly matching layers were imposed at upper and lower boundaries to absorb the outgoing electromagnetic waves without producing significant reflections back into the simulation domain. The simulation of two-dimensional ordered $\mathrm{Cu}$ nanoparticle arrays was performed using periodic boundary conditions. The cell size and the time step used in discretization of the space domain and time domain were $1 \mathrm{~nm} \times 1 \mathrm{~nm} \times 1 \mathrm{~nm}$ and $1.9 \times 10^{-18} \mathrm{~s}$, respectively. A planewave with an $x$-directed electric field was launched from the glass substrate along the positive $z$-direction.

Figure 6 presents the field distributions of the $x-y$ plane at the resonant wavelength of TP mode. The strengths of electric field and magnetic field both increased when the gap was changed from $50 \mathrm{~nm}$ to $30 \mathrm{~nm}$. The incident light is trapped and redistributed in space to effectively generate excitons at the interface between the TPA and $\mathrm{Cu}$ nanoparticles.
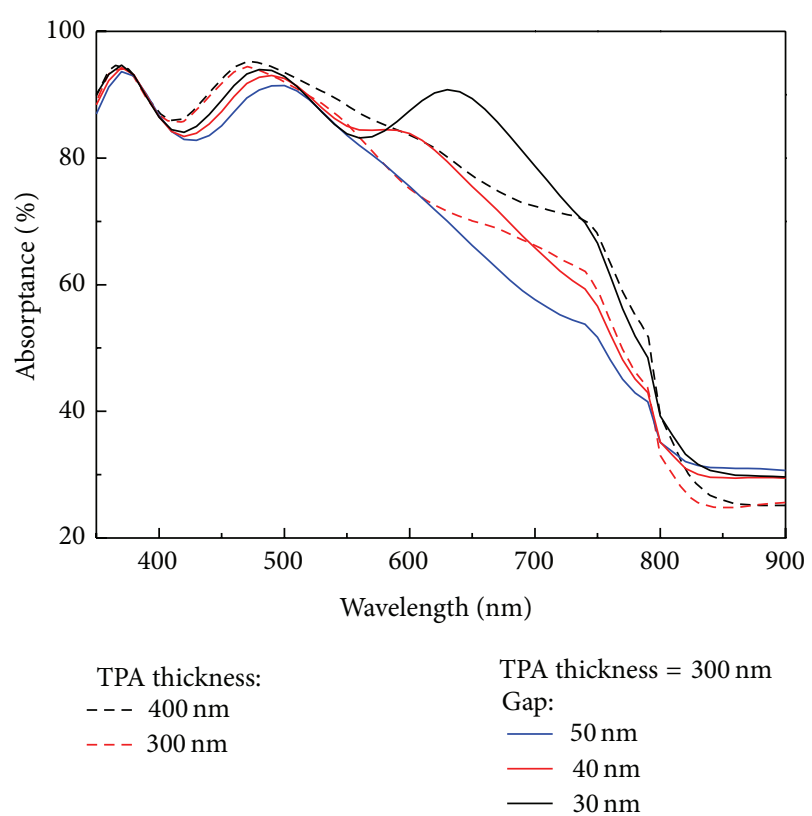

FIGURE 5: The red (black) dashed line indicated the absorptance of the TPA/ITO/glass when the TPA thickness is equal to $300 \mathrm{~nm}$ (400 nm). The blue, red, and black lines show the absorptance of the TPA/ITO/glass with the $\mathrm{Cu}$ nanoplasmonic structure when the long axis of the $\mathrm{Cu}$ nanoparticles is $150 \mathrm{~nm}$.

Conceptually, the excitons can be dissociated at the interface between TPA and $\mathrm{Cu}$ nanoparticles because the HOMO level $(-5.4 \mathrm{eV})$ [3] of the TPA is lower than the Fermi level of $\mathrm{Cu}(-4.94 \mathrm{eV})$ [12]. Therefore, the localized field distribution benefits the exciton dissociation.

Figure 7 presents the electric field distributions of $\mathrm{Cu}$ nanoplasmonic structures embedded in the TPA film on the $x-z$ plane. The electric fields (excitons) are localized (generated) around the lower surface of $\mathrm{Cu}$ nanoparticles. Therefore, the excitons are dissociated mostly from the lower surface of the $\mathrm{Cu}$ nanoparticles. After exciton dissociation, the holes (electrons) can propagate along the $\mathrm{Cu}$ (TPA) to the anode (cathode) electrode. In such cases, carrier recombination can be reduced.

\section{Conclusions}

In conclusion, we have assessed the optical effects of $\mathrm{Cu}$ nanoplasmonic structure embedded in triiodide perovskite absorber (TPA). The refractive index and absorption coefficient of TPA were obtained by fitting the transmittance spectrum of TPA/PEDOT:PSS/ITO/glass using transfer matrix method. $\mathrm{Cu}$ nanoplasmonic structures could reduce the TPA thickness from $400 \mathrm{~nm}$ to $300 \mathrm{~nm}$ while keeping the absorption strength. The 3D finite-difference time-domain method was used to observe the distribution of the electric field (generated excitons). The electric field is redistributed at the interface between the TPA and $\mathrm{Cu}$ nanoparticles, which benefits the exciton dissociation and carrier transport. 


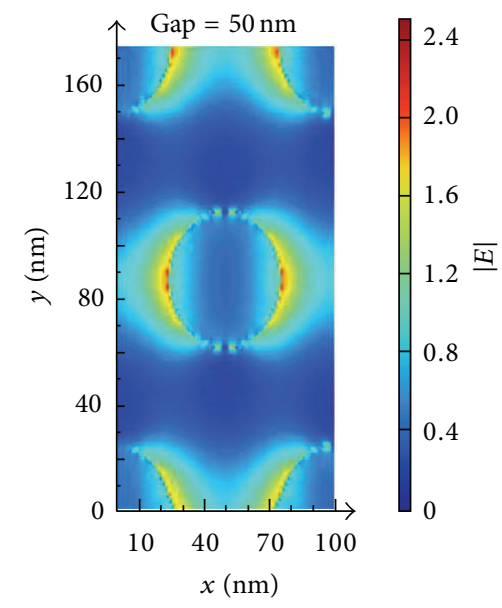

(a)

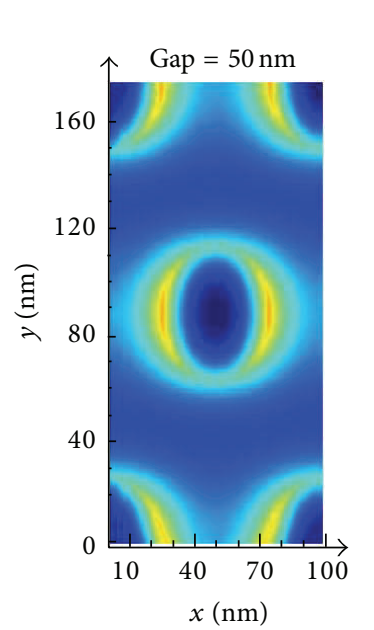

(d)

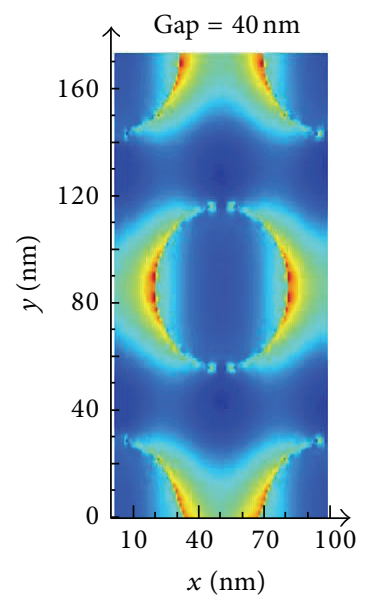

(b)
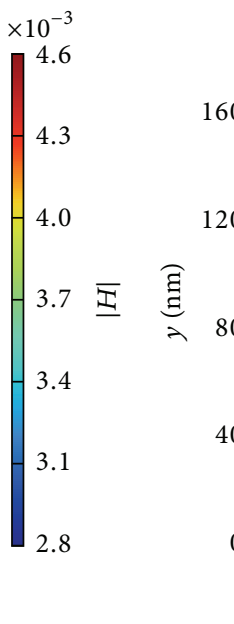

${ }_{0}^{40}$

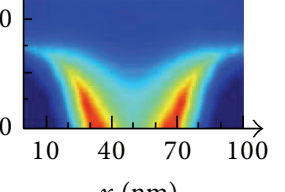

(e)
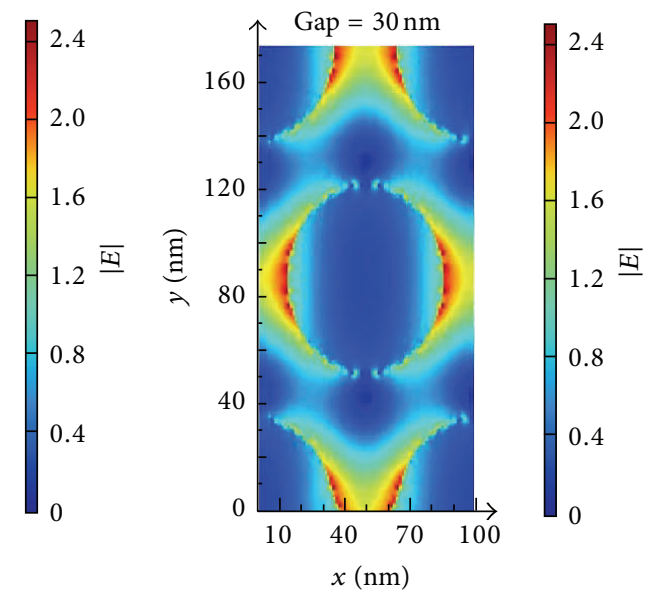

(c)
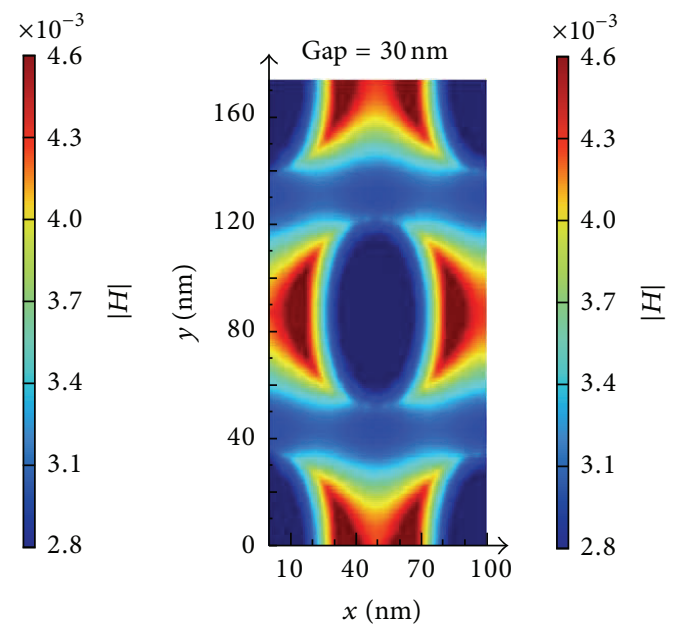

(f)

Figure 6: (a), (b), and (c) ((d), (e), and (f)) are the electric (magnetic) field distributions of the $x-y$ plane for different gap sizes.

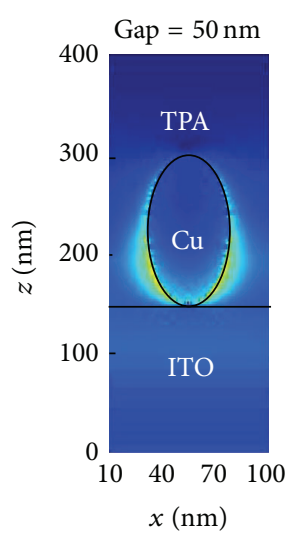

(a)

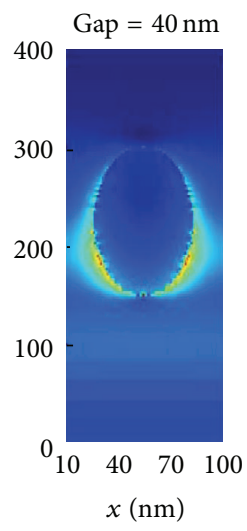

(b)

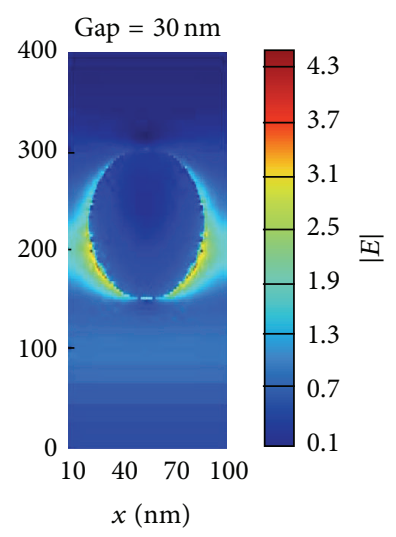

(c)

Figure 7: (a), (b), and (c) are the electric field distributions of the $x$ - $z$ plane for different gap sizes. 


\section{Conflict of Interests}

The authors declare that there is no conflict of interests regarding the publication of this paper.

\section{Acknowledgment}

This work was supported by the National Science Council under Grant NSC 101-2731-M-008-002-MY3.

\section{References}

[1] J. Burschka, N. Pellet, S.-J. Moon et al., "Sequential deposition as a route to high-performance perovskite-sensitized solar cells," Nature, vol. 499, no. 7458, pp. 316-319, 2013.

[2] D. Liu and T. L. Kelly, "Perovskite solar cells with a planar heterojunction structure prepared using room-temperature solution processing techniques," Nature Photonics, vol. 8, pp. 133-138, 2014.

[3] G. Xing, N. Mathews, S. Sun et al., "Long-range balanced electron- and hole-transport lengths in organic-inorganic $\mathrm{CH}_{3} \mathrm{NH}_{3} \mathrm{PbI}_{3}$," Science, vol. 342, pp. 344-347, 2013.

[4] S. D. Stranks, G. E. Eperon, G. Grancini et al., "Electron-hole diffusion lengths exceeding 1 micrometer in an organometal trihalide perovskite absorber," Science, vol. 342, pp. 341-344, 2013.

[5] V. D'Innocenzo, G. Grancini, J. P. Marcelo et al., "Excitons versus free charges in organo-lead tri-halide perovskites," Nature Communications, vol. 5, article 3586, 2014.

[6] S. de Wolf, J. Holovsky, S.-J. Moon et al., "Organometallic halide perovskites: sharp optical absorption edge and its relation to photovoltaic performance," The Journal of Physical Chemistry Letters, vol. 5, no. 6, pp. 1035-1039, 2014.

[7] N.-G. Park, "Organometal perovskite light absorbers toward a $20 \%$ efficiency low-cost solid-state mesoscopic solar cell," The Journal of Physical Chemistry Letters, vol. 4, no. 15, pp. 24232429, 2013.

[8] N. F. Fahim, B. Jia, Z. Shi, and M. Gu, "Simultaneous broadband light trapping and fill factor enhancement in crystalline silicon solar cells induced by Ag nanoparticles and nanoshells," Optics Express, vol. 20, no. 19, pp. A694-A705, 2012.

[9] S. H. Chang, "Modeling and design of $\mathrm{Ag}, \mathrm{Au}$, and $\mathrm{Cu}$ nanoplasmonic structures for enhancing the absorption of P3HT:PCBMbased photovoltaics," IEEE Photonics Journal, vol. 5, no. 3, Article ID 4800509, 2013.

[10] S. H. Chang, B.-Y. Lin, T.-Y. Cheng, and J.-K. Wang, "Unraveling electromagnetic resonance of two-dimensional ordered nanoparticle arrays with a dipole-coupling model," Physica Status Solidi: Rapid Research Letters, vol. 4, no. 10, pp. 259-261, 2010.

[11] A. D. Rakić, A. B. Djurišić, J. M. Elazar, and M. L. Majewski, "Optical properties of metallic films for vertical-cavity optoelectronic devices," Applied Optics, vol. 37, no. 22, pp. 5271-5283, 1998.

[12] P. O. Gartland, S. Berge, and B. J. Slagsvold, "Photoelectric work function of a copper single crystal for the (100), (110), (111), and (112) faces," Physical Review Letters, vol. 28, no. 12, pp. 738-739, 1972. 

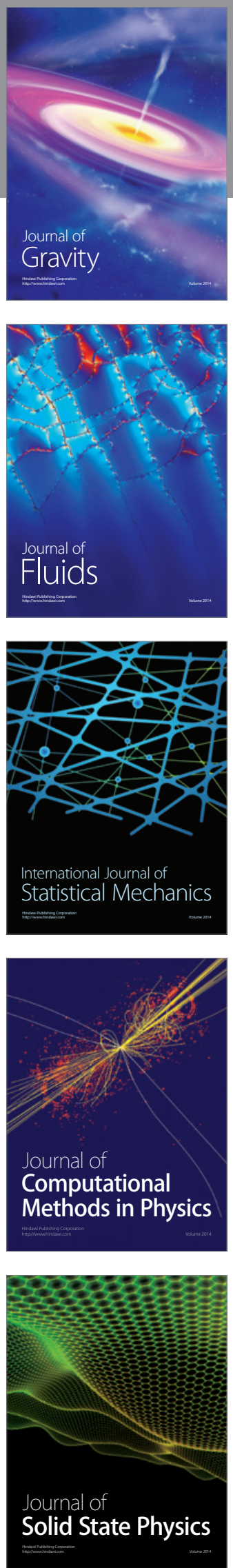

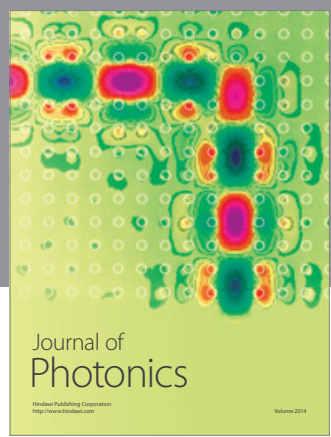

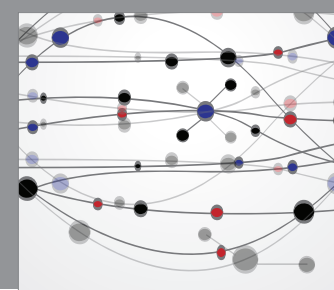

The Scientific World Journal

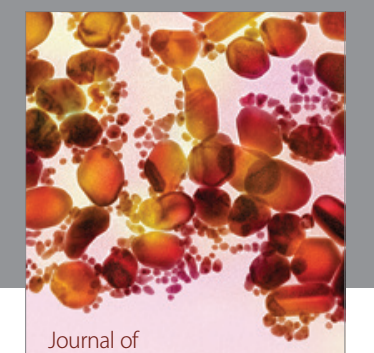

Soft Matter
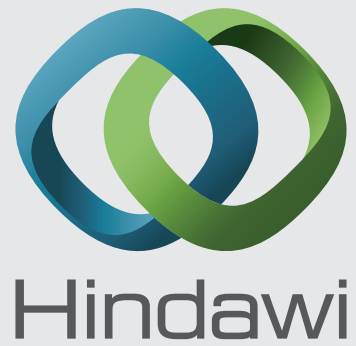

Submit your manuscripts at

http://www.hindawi.com
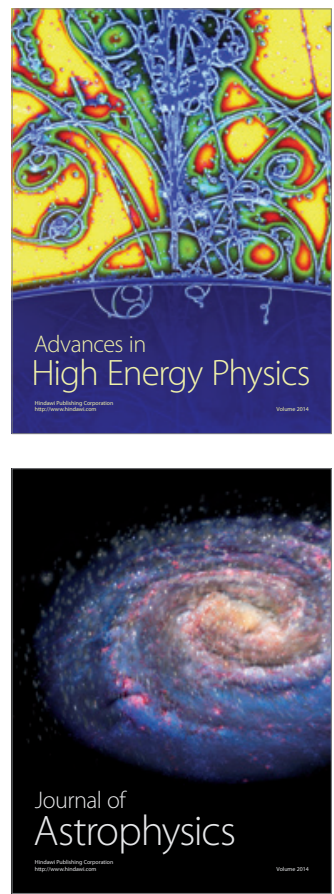
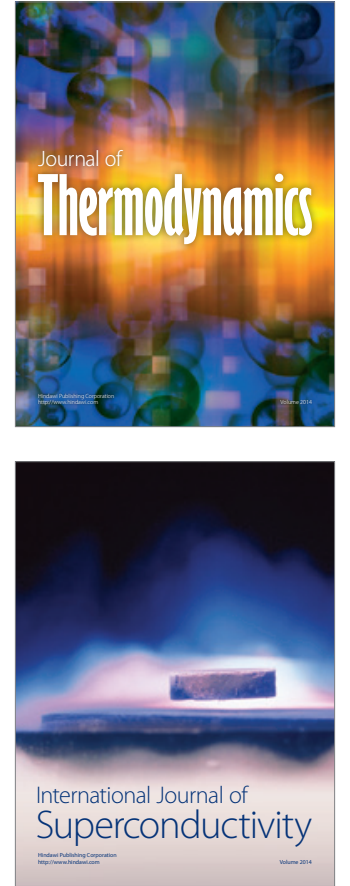
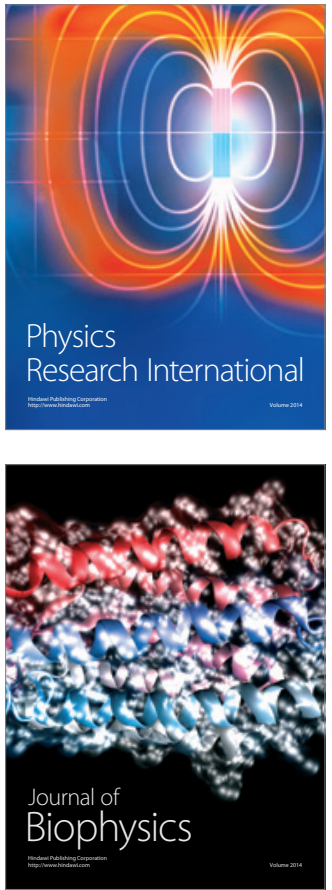
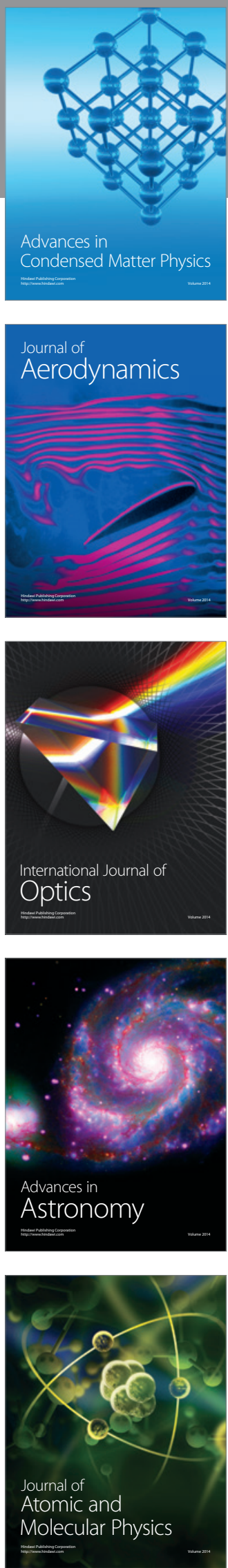